Publ. Astron. Soc. Aust., 1997, 14, 8-10

\title{
TTF Survey of Galaxy Populations
}

\author{
Heath Jones ${ }^{1}$ and Joss Bland-Hawthorn ${ }^{2}$ \\ 1 Mount Stromlo \& Siding Spring Observatories, \\ Private Bag, Weston Creek PO, ACT 2611, Australia \\ dhj@mso.anu.edu.au \\ 2 Anglo-Australian Observatory, PO Box 296, Epping, NSW 2121, Australia \\ jbh@aaoepp.aao.gov.au \\ Received 1996 September 12, accepted 1996 October 18
}

\begin{abstract}
The TAURUS Tunable Filter (TTF) affords a new approach to observational cosmology, allowing a wide field $(10$ arcmin) to be imaged monochromatically in contiguous wavelength intervals (6-60 $\AA$ bandpass) over the $\mathrm{R}$ and I bands. In a $200 \mathrm{~s}$ exposure with the AAT, the TTF can detect $\mathrm{H} \alpha$ emission powered by star formation rates as low as $0 \cdot 1 M_{\odot} \mathrm{yr}^{-1}$ at $z=0.08$ and $1 M_{\odot} \mathrm{yr}^{-1}$ at $z=0.24$ in $2 \operatorname{arcsec}$ seeing (cf. $0 \cdot 26 M_{\odot} \mathrm{yr}^{-1}$ for the LMC). In this paper we describe an emission-line survey currently under way using the TTF on the AAT to detect redshifted $\mathrm{H} \alpha$ over the ranges $z=0 \cdot 06-0 \cdot 1$ and $z=0 \cdot 22-0 \cdot 26$. Such detections will be of timely interest to the Southern H I Sky Survey which is motivated along similar lines.
\end{abstract}

Keywords: cosmology: observations - galaxies: surveys - galaxies: emission-line - instrumentation: Fabry-Perot

\section{Introduction}

The TAURUS Tunable Filter (TTF) is a new facility at the AAO that allows fully monochromatic narrowband imaging over an arbitrary bandpass (6$60 \AA \mathrm{FWHM}$ ) at $\mathrm{R}$ and I bands. This instrument combines the flexibility of an ordinary Fabry-Perot interference filter to scan in wavelength with the widefield monochromaticity of fixed narrowband interference filters. Unlike an ordinary Fabry-Perot. however, the TTF works at orders low enough that a single interference ring covers almost all of the detector area.

The strength of the TTF is its unique ability to detect faint emission-line sources over a useful area on the sky. These are emission-line sources that would otherwise be lost within background sky noise on broadband images. We intend to exploit this facility as a means of probing for faint field and cluster emission-line galaxies (ELGs) at cosmologically interesting distances.

\section{Survey Motivation}

One of the current problems of observational cosmology is the inability to reconcile the large numbers of blue galaxies seen at faint magnitudes [see Koo \& Kron (1992) for a review, and more recent work by Lilly, Cowie \& Gardner (1991) and Metcalfe et al. (1995)] with the results of recent spectroscopic redshift surveys (Ellis et al. 1996, and references therein). These surveys $(B \lesssim 24)$ indicate that the majority of these objects lie at modest redshift $(z \sim 0 \cdot 3)$ rather than the high redshifts originally expected. While simple no-evolution (NE) models of the universe are able to account for the slope of the observed redshift distribution, the number counts of these faint blue galaxies (FBGs) to faint limits $(B \sim 28)$ are well in excess of those predicted by NE models. Furthermore, it appears that the number count data are more easily modelled with the use of a non-zero cosmological constant and $q_{0} \neq 0 \cdot 5$, both of which are disfavoured options from a theoretical standpoint (Schade \& Ferguson 1994).

Ellis et al. (1996) claim that the excess population of galaxies at $z \sim 0.3$ is primarily due to a separate population of star-forming galaxies characterised by broad [O II] equivalent widths, a suggestion first made by Broadhurst, Ellis \& Shanks (1988) and later confirmed by Broadhurst, Ellis \& Glazebrook (1992) as the spectroscopic studies increased in depth. No such equivalent population has yet been detected locally $(z \lesssim 0 \cdot 1)$. This is a crucial issue in the interpretation of the excess FBG counts, for if local galaxy samples are incomplete (e.g. due to surface brightness selection effects) then an artificial excess in the galaxy counts will appear at higher redshift (Schade \& Ferguson 1994). The TTF Field Galaxy Survey aims to place stronger constraints on galaxy SFRs and luminosities both at $z \sim 0.25$ where the FBG excess is observed, and at $z \lesssim 0 \cdot 1$ where an equivalent local population has long been suspected but not observed. 


\section{Observing Strategy}

We are undertaking a volume-limited survey for $\mathrm{H} \alpha$ line emission from galaxies within two intervals of redshift: $0.064 \leq z \leq 0.101$ (interval A) and $0.225 \leq z \leq 0.259$ (interval $\mathrm{B}$ ), using the 10 arcmin field of the TTF. Although the primary target of the survey is $\mathrm{H} \alpha$ emission, the survey will also be sensitive to emission from redshifted [O II], $\mathrm{H} \beta$, [O III] and possibly Ly $\alpha$. Table 1 summarises the scanning parameters of the TTF for each interval.

The intrinsic $\mathrm{H} \alpha$ line strength in a quiescent galaxy can be related to the current rate of massive star formation present (Kennicutt 1983). Figure 1a shows apparent $\mathrm{H} \alpha$ luminosities, $F_{\mathrm{H} \alpha}$, as a function of redshift for galaxies with star formation rates (SFRs) between 0.01 and $10 M_{\odot} \mathrm{yr}^{-1}$. We have assumed $H_{0}=75 \mathrm{~km} \mathrm{~s}^{-1} \mathrm{Mpc}^{-1}, \Omega_{0}=0.2$ and $\Lambda_{0}=0$ throughout. Figure $1 \mathrm{~b}$ shows the signal-tonoise ratio $(S / N)$ per pixel that we expect to attain using the TTF for galaxies with SFRs between $0 \cdot 1$ and $10 M_{\odot} \mathrm{yr}^{-1}$. We have assumed emission from galaxies at redshifts of 0.08 (interval A; solid curve) and $0 \cdot 24$ (interval B; dashed curve). Typical values of dark current, read noise and sky background during bright time have been used with a combined AAT/TTF/CCD efficiency of $17 \%$. Since a typical star-forming region in an LMC-type dwarf is about $300 \mathrm{pc}$ in diameter, we expect the emission-line regions to be spatially unresolved and so Figure $1 b$ assumes 2 arcsec seeing.

As Figure 1b shows, SFRs as low as $0 \cdot 1 M_{\odot} \mathrm{yr}^{-1}$ at $z=0.08$ and $1 M_{\odot} \mathrm{yr}^{-1}$ at $z=0.24$ can yield a $3 \sigma$ detection in only $130 \mathrm{~s}$ of exposure time. By way of comparison, a low-activity Sb spiral such as the Milky Way has a SFR of $\sim 5 M_{\odot} \mathrm{yr}^{-1}$ (Smith, Biermann \& Mezger 1978) while the LMC has a rate of $0.26 M_{\odot} \mathrm{yr}^{-1}$ (Kennicutt et al. 1995). We have settled upon an exposure time of $200 \mathrm{~s}$ per slice as a compromise between flux limit and sky coverage. Exposures of this duration allow us to obtain $\mathrm{H} \alpha$ fluxes to $\sim 10^{-15} \mathrm{erg} \mathrm{cm}^{-2} \mathrm{~s}^{-1}\left(\sim 10^{-26}\right.$ $\mathrm{W} \mathrm{m}^{-2}$ ) as Figure 1a shows.

\section{Scientific Returns}

The TTF Field Galaxy Survey aims to cover $0.56 \square^{\circ}$ on the sky $\left(\sim 13200 \mathrm{Mpc}^{3}\right)$, the returns from which we expect to be numerous. First, the survey promises unprecedented detection of $\mathrm{H} \alpha$ emission in the separate star-forming galaxy population at $z \sim 0 \cdot 3$, thereby providing a quantifiable and independent confirmation of its extent compared to the [O II] detections of recent spectroscopic surveys. Second, we expect to extend the current limits for detection of distant emission-line galaxies, due to our low background advantage and high line/continuum ratio compared to those in broadband surveys. Finally, at $z \sim 0.1$ we are probing for $\mathrm{H} \alpha$ emission

Table 1. Scanning parameters used in the survey

\begin{tabular}{cccccc}
\hline Interval & $\begin{array}{c}\text { Wavelength } \\
\text { range }(\AA)\end{array}$ & $\begin{array}{c}\mathrm{H} \alpha \\
\text { redshift }\end{array}$ & $\begin{array}{c}\text { No. of } \\
\text { slices }\end{array}$ & $\begin{array}{c}\text { Scan } \\
\text { step }(\AA)\end{array}$ & $\begin{array}{r}\text { FWHM* }^{*} \\
\left(\mathrm{~km} \mathrm{~s}^{-1}\right)\end{array}$ \\
\hline $\mathrm{A}$ & $\lambda \lambda 6980-7227$ & $0 \cdot 064 \leq z \leq 0 \cdot 101$ & 27 & $9 \cdot 5$ & 311 \\
$\mathrm{~B}$ & $\lambda \lambda 8040-8264$ & $0 \cdot 225 \leq z \leq 0 \cdot 259$ & 19 & $12 \cdot 5$ & 369 \\
\hline
\end{tabular}

* Resolution kept fixed during scanning.
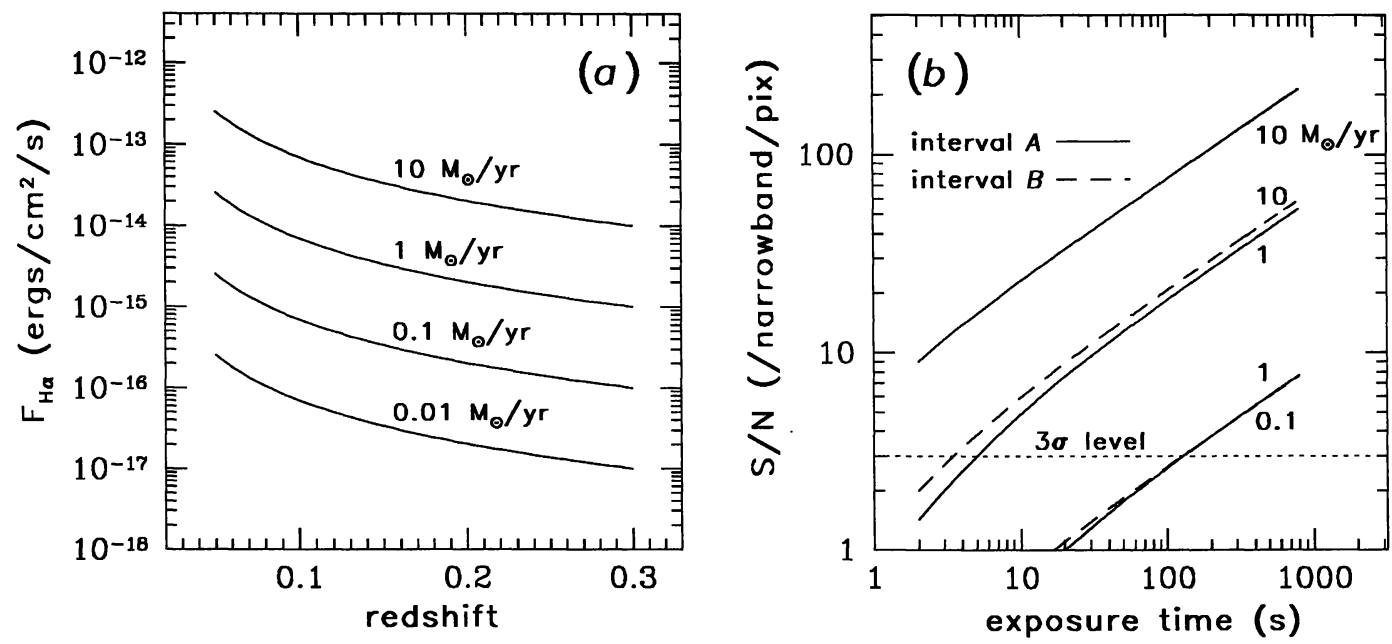

Figure 1-Calculations used to optimise survey coverage. In (b) the solid and dashed lines correspond to redshift intervals A $(0.064 \leq z \leq 0.101)$ and $B(0.225 \leq z \leq 0.259)$ respectively. See text for details. 
from local counterparts to the FBGs, the results of which will provide much stronger constraints on the star-formation rates found at $z \lessgtr 0 \cdot 1$ than have previously been possible.

Broadhurst, T. J., Ellis, R. S., \& Shanks, T. 1988, MNRAS, 235,827

Broadhurst, T. J., Ellis, R. S., \& Glazebrook, K. 1992, Nature, 355,55
Ellis, R. S., Colless, M. M., Broadhurst, T. J., Heyl, J., \& Glazebrook, K. 1996, MNRAS, 280, 235

Kennicutt, R. C. 1983, ApJ, 272, 54

Kennicutt, R. C., Bresolin, F., Bomans, D. J., Bothun, G. D., \& Thompson, I. B. 1995, AJ, 109, 594

Koo, D., \& Kron, R. 1992, ARA\&A, 30, 613

Lilly, S. J., Cowie, L. L., \& Gardner, J. P. 1991, ApJ, 369, 79

Metcalfe, N., Shanks, T., Fong, R., \& Roche, N. 1995, MNRAS, 273, 257

Schade, D. J., \& Ferguson, H. C. 1994, MNRAS, 267, 889

Smith, L. F., Biermann, P., \& Mezger, P. G. 1978, AA, 66, 65 